\title{
Control design of a de-weighting upper-limb exoskeleton: extended-based fuzzy
}

\author{
Siti Khadijah Ali ${ }^{1}$ and M.Osman Tokhi ${ }^{2}$ \\ ${ }^{1}$ Department of Multimedia, Universiti Putra Malaysia, Malaysia \\ ${ }^{2}$ Department of Electrical and Electronic Engineering, London South Bank University, United Kingdom
}

\begin{tabular}{l} 
Article Info \\
\hline Article historys: \\
Received Dec 31, 2018 \\
Revised Jan 27, 2019 \\
Accepted Feb 22, 2019 \\
\hline Keywords: \\
Control approach \\
Extended-based fuzzy control \\
Fuzzy logic control \\
Muscle fatigue \\
Upper-limb exoskeleton \\
\hline
\end{tabular}

\begin{abstract}
One of the most common issues to human is fatigue. A technology known as exoskeleton has been identified as one of the solutions to address this issue. However, there are two issues that need to be solved. One of them is the control approach. Hence, the main aim of this work, is to investigate the control design for upper-limb exoskeleton. An extended based fuzzy control is proposed to observe the effectiveness of the exoskeleton in dealing with human with different strength. Three conditions of human strength were applied. PID was used for a comparison purpose. It is shown that with the proposed control approach, the exoskeleton can assist human to achieve the desired trajectory accurately with a minimal amount of torque required.
\end{abstract}

Copyright $(2019$ Institute of Advanced Engineering and Science. All rights reserved.

\section{Corresponding Author:}

Siti Khadijah Ali,

Department of Multimedia,

Faculty of Computer Science and Information Technology,

Universiti Putra Malaysia, Malaysia.

Email: ctkhadijah@upm.edu.my

\section{INTRODUCTION}

Fatigue is an important issue for human, especially to professional athletics, mining industries and manufacturing. Fatigue could be defined as a condition occur to human when the motion strength is lower than the strength needed to perform an exercise or task [1], [2]. There are factors that could contribute to fatigue such as manual handling of objects, heavy physical activities, repetitive work and improper body posture. These activities require muscles to contract high and low and require muscle to perform sustained or intermittent actions. In addition, there are certain activities that require muscle to stay either static or dynamic.

Muscle fatigue could case reduction of power, lead to discomfort and pain. Furthermore, in a worst condition, muscle fatigue could lead to cumulative trauma disorder (CTD) and musculoskeletal disorder (MSD) [3], [7]. According to [8] the major forms of MSD in work-related morbidity include upper-limb disorders and low back pain. This problem could affect all stage of people, from the worker to the organization and society, especially in financial cost. For example, [9] reported that about $67 \%$ of all work-related injuries were due to upper-limb disorder. The cost to cover due to this problem is about $\$ 110$ billion dollars annually for medical expenses, lost productivity and wages.

Researchers have developed several types of fatigue model to identify the occurrence of the fatigue, hence, could reduce the potential to get MSDs. These models have been used widely in ergonomics research for industrial applications [6], [10]-[14]. Apart from providing the fatigue model to some industrial applications to solve fatigue, there is another solution to this issue; exoskeleton. Several works have been done to investigate the capability of the exoskeleton in supporting and assisting human in performing tasks while fatigue is occurred [15]-[18]. 
Exoskeleton could be worn on human upper-limb, lower-limb or on the whole body. From literature, exoskeleton could be categorized based on the application, human-interaction, mechanical design and control approach. Generally, there are two important issues in developing the exoskeleton: the design and the control system [5], [6], [8], [13], 14], [18]-[20]. It is essential to ensure that the exoskeleton is ergonomics to human, and the control approach is effective, to ensure that the exoskeleton could be operated harmoniously with human limb. Currently, in most research relating to the development of control approach for an exoskeleton, human activities will be stopped when fatigue occurs.

There are several ways to ensure that the exoskeleton is moving smoothly with human motion, and the common control strategies are position and impedance control [21], [22]. In addition, several techniques have been proposed to de-weight the exoskeleton by compensating the gravity and friction effects [22], [23]. Currently, more researchers are focusing on the advanced control. For instance, implementing PD control to control passive mode movement and implement neuro-fuzzy based biological controller for assisting active move movement [24]. One of the ways to detect human signals or strengths is by using electromyography (EMG). PID is one of the most common controllers that has been used integrated with EMG signals and button count.

A research proposed a cascade control approach [17]. The cascade control approach consists of iterative learning control (ILC) and the fatigue is considered as a disturbance to the muscle model. Although the results from this approach shows promising outcome, however, several needs still need to be done especially, for the usage of ILC and the fatigue model itself [17]. [25] enhanced [17] by improving the muscle and fatigue model. However, these two works are focusing only on the rehabilitation application [25]. Hence, in this work, the focus is to investigate the effectiveness of the control approach of the exoskeleton in assisting fatigue people while doing work in domestic environment.

\section{RESEARCH METHOD}

\subsection{System Description}

There are three software tools used in this work: SolidWorks, Matlab and Simmechanics. SolidWorks is a computer aided deisgn (CAD software, that allows users to design the complex 3D realistic models. In addition, SolidWorks could also be imported to other software such as VisualNastran and SimMechanics. SimMechanics is a part of Simulink Toolbox provided by the MATLAB. It is chosen due to its ability to allow users to model mechanical systems. SimMechnics also allows for animation or simulation purpose. In addition, the performance of developed control mechanism also could be evaluated by using SimMechanics. The humanoid is developed to represent the human-like physical system in terms of measurements of mass, height and length Figure 1. Table 1 shows the measurements for the length and mass of human model [26].

The design of the exoskeleton is inspired by TitanArm [27] due to its simplicity, capable of powered use and data transmission in a mobile fashion. The exoskeleton was designed with aluminum to provide the exoskeleton structure with a relatively light weight. This is because aluminum is a low-density material and has reasonable strength characteristics. The exoskeleton is worn on the lateral side of the upper limb to prove naturalistic movements of the shoulder, elbow and wrist. The exoskeleton used in this research consists of four joints: shoulder abduction-adduction, shoulder internal-external, shoulder flexion-extension and elbow flexionextension. Then, the design of human-exoskeleton is, imported to SimMechanics for control system evaluation purpose.

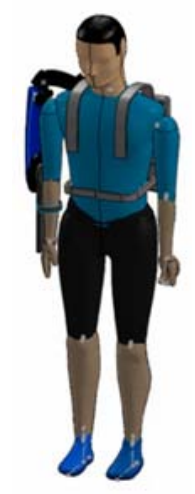

Figure 1. The exoskeleton is attached parallel to human 
Table 1. Physical model parameters

\begin{tabular}{ccc}
\hline Segment & Length $(\mathrm{cm})$ & Mass $(\mathrm{kg})$ \\
\hline Head & 22 & 3 \\
Neck & 8.8 & 1.085 \\
Trunk & 49 & 34 \\
Pelvis & 5 & 4.687 \\
Upper arm & 31.32 & 2.17 \\
Lower arm & 28.08 & 1.30 \\
Hand & 19.42 & 0.49 \\
Thigh & 41.6 & 7 \\
Calf & 41.8 & 3.26 \\
Foot & 25.8 & 1.015 \\
\hline
\end{tabular}

\subsection{Kinematics of the designed exoskeleton}

Kinematics and dynamics are the two terms mostly used in robotic research. Kinematics is defined as the study of motion without considering the force, torque and moment. Two groups of kinematics are, forward kinematics and inverse kinematics. Forward kinematics is a process of obtaining the end-effector position when the angles of the joints are given. Inverse kinematics is a process of calculating the angles of the joints when the end-effector position is given. This may be described as in Figure 2.

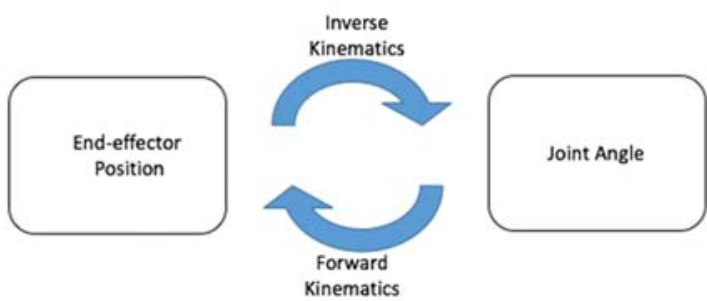

Figure 2. Forward and inverse kinematics

Forward kinematics: The Denavit-Hartenberg (DH) convention is used to obtain forward kinematics. The DH notation is chosen because it allows composing coordinate transformation into one homogenous transformation matrix. The homogenous transformation matrix provides the relative position and orientation of two consecutive frames. This information is used to connect two consecutive frames. The two consecutive frames could be described as i-1 and i. As shown in Figure 3 (a), the base frame for the exoskeleton is denoted as $\mathrm{O}_{0}$. The $\mathrm{O}_{0}$ also represents the shoulder adduction/abduction motion. The $\mathrm{O}_{1}, \mathrm{O}_{2}$ and $\mathrm{O}_{3}$ represent shoulder internal/external motion, shoulder extension/flexion and elbow extension/flexion respectively. $\mathrm{O}_{4}$ represents the end-point of the exoskeleton.

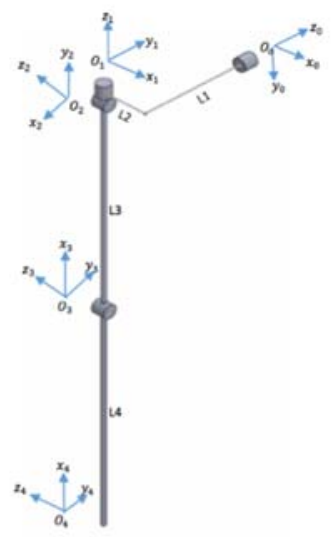

(a)

\begin{tabular}{|c|c|c|c|c|}
\hline Frame & $\theta_{i}$ & $\alpha_{i}$ & $d_{i}$ & $a_{i}$ \\
\hline 1 & $\theta_{1}$ & $\frac{\pi}{2}$ & $L_{1}$ & $L_{2}$ \\
\hline 2 & $\theta_{2}-\frac{\pi}{2}$ & $\frac{\pi}{2}$ & 0 & 0 \\
\hline 3 & $\theta_{3}+\frac{\pi}{2}$ & 0 & 0 & $L_{3}$ \\
\hline 4 & $\theta_{4}$ & 0 & 0 & $L_{4}$ \\
\hline
\end{tabular}

(b)

Figure 3. (a) Schematic diagram (b) Denavit-Hartenberg table 
In Figure 3 (b), the DH table consists of four parameters: $\theta_{i}, \alpha_{i}, a_{i}, d_{i}$ where

a) $\theta_{i}$ represents the angle between $X_{i-1}$ and $X_{i}$ which is measured around $Z_{i-1}$

b) $\alpha_{i}$ represents the angle between $Z_{i-1}$ and $Z_{i}$ which is measured around $X_{i}$

c) $a_{i}$ represents the distance along $X_{i}$ from $O_{i}$ to the intersection of the axis $X_{i}$ and $Z_{i-1}$

d) $d_{i}$ represents the distance along $Z_{i-1}$ from $O_{i-1}$ to the intersection of $X_{i}$ and $Z_{i-1}$ axes

There are three important steps to determine the frame. The steps are as follows:

a) Determine the origin of the axes $\left(O_{i}\right)$.

b) Determine the $\mathrm{z}$-axis of each frame. The $\mathrm{z}$-axis is determined based on the direction of motion for each joint.

c) Determine the $x$-axis of each frame. There are three rules to determine the $x$-axis. The rules are basically based on the position of the $Z_{i-1}$ and $Z_{i}$. The rules are presented as follows:

a. If the $Z_{i-1}$ and $Z_{i}$ are nor co-planar, there exists a unique line segment perpendicular to both $Z_{i-1}$ and $Z_{i}$. This line defines $\mathrm{x}$-axis for frame $i$.

b. If the $Z_{i-1}$ and $Z_{i}$ are parallel, there exists an infinite line segment perpendicular to $Z_{i-1}$ and $Z_{i}$, and the $\mathrm{x}$-axis for frame $i$ can be chosen from one of these lines. There are two options for choosing the direction of the $X_{i}$ - axis:

i. The $X_{i}$ - axis could be pointing to $Z_{i-1}$

ii. The $X_{i}$ - axis could be not pointing to $Z_{i-1}$

d) Since, the $Z_{i-1}$ and $Z_{i}$ are parallel, hence, the $d_{i}=0$ and $\alpha_{i}=0$.

a. If the $Z_{i-1}$ and $Z_{i}$ are intersection, the $X_{i}$ is chosen normal to the plane formed by $Z_{i-1}$ and $Z_{i}$. For this case, the $a_{i}=0$.

e) Determine the $y$-axis of each frame. Once, the $x$ - and $z$-axis has been identified, the $y$-axes will be determined by using right-hand rule.

Inverse kinematics: In this work, the inverse kinematics is obtained by using closed-form solution, specifically the algebraic approach is chosen due to uncomplex model of the exoskeleton. The exoskeleton consists of four joints Figure 3(a): shoulder abduction-adduction $\left(\theta_{1}\right)$, shoulder internal-external $\left(\theta_{2}\right)$, shoulder extension-flexion $\left(\theta_{3}\right)$ and elbow extension-flexion $\left(\theta_{4}\right)$. However, in this work, the shoulder internal-external is restricted between $-60^{0}$ to $60^{\circ}$. It is assumed that $\theta_{2}$, is known. Hence $\theta_{3}$ could be obtained as follows:

$$
\pm \theta_{3}=\sin ^{-1} M, M=\frac{z_{3}-L_{1}}{L_{3} \cos \theta_{2}}
$$

As presented in (1), there are two possible values of $\theta_{3}$. To determine the sign of the $\theta_{i}$, an algorithm based on unit circle is used. Unit circle can be seen on Figure 4.

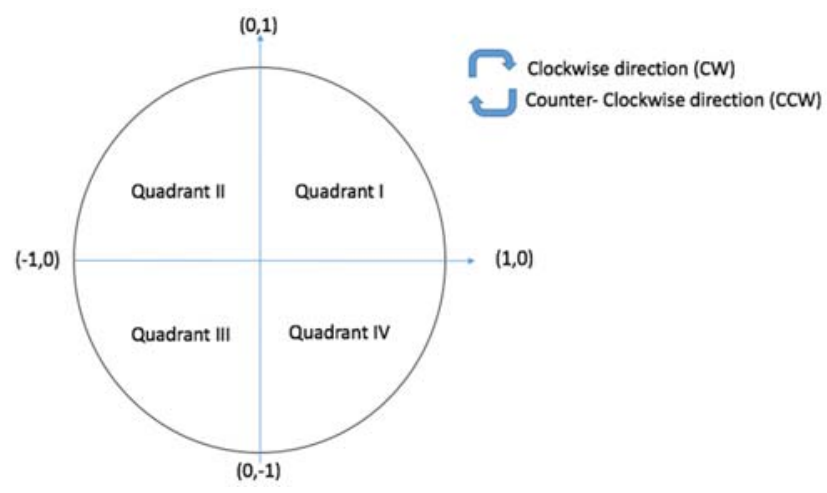

Figure 4. Unit circle

The unit circle consists of length with radius 1 and is divided into four quadrants. For the case of $\theta_{3}$, the range is between 0 to $\pi$, hence the possible values of $\theta_{3}$ exists in Quadrant I or II. The algorithm for $\theta_{3}$ as shown in Table 2.

Indo. J. Elec. Eng. \& Inf, Vol. 7, No. 1, March 2019: 1 - 14 
Table 2. Complete algorithm for $\theta_{3}$

\begin{tabular}{lc}
\hline No & \multicolumn{1}{c}{ Algorithm } \\
1. & If input $\geq 0$ \\
2. & If $\theta_{3} \geq$ input \\
3. & $\theta_{3}=\theta_{3}$ \\
4. & Else \\
5. & $\theta_{3}=\pi-\theta_{3}$ \\
6. & \\
7. & Else \\
8. & If $\theta_{3}==$ input \\
9. & $\theta_{3}=\theta_{3}$ \\
10. & Else \\
11 & $\theta_{3}=-\left(\pi+\theta_{3}\right)$ \\
\hline
\end{tabular}

Figure 5 shows the position of the $\theta_{4}$. To obtain $\theta_{a}$, cosine law is used. $\theta_{4}$ is an angle which presenting the movement of the elbow from the initial position (dot lines) to some specific position Figure 5 . The L, could be obtained as:

$$
L=\sqrt{\left(x_{1}-y_{1}\right)^{2}+\left(x_{2}-y_{2}\right)^{2}+\left(x_{3}-y_{3}\right)^{2}}
$$

The cosine law is presented as follows:

$$
L^{2}=U A^{2}+L A^{2}-2 * U A * L A * \cos \theta_{a}
$$

Thus, $\theta_{a}$ is calculated as:

$\pm \theta_{a}=\cos ^{-1} \frac{U A^{2}+L A^{2}-L^{2}}{2 * U A * L A}$

Then, $\theta_{4}$ is obtained as:

$$
\theta_{4}=\pi-\left( \pm \theta_{a}\right)
$$

Note that $\theta_{4}$ is restricted to $[0, \pi]$, hence the possible values exist in Quadrant I and Quadrant II. A condition statement is applied to obtain the final value of $\theta_{4}$ in (5). The condition statement is

\begin{tabular}{|c|c|}
\hline No. & Algorithm \\
\hline 1. & If input $\geq 0$ \\
\hline 2. & $\theta_{4}=\theta_{4}$ \\
\hline 3. & Else \\
\hline 4. & $\theta_{4}=-\theta_{4}$ \\
\hline
\end{tabular}
shown in Table 3.

Table 3. Complete algorithm for $\theta_{4}$

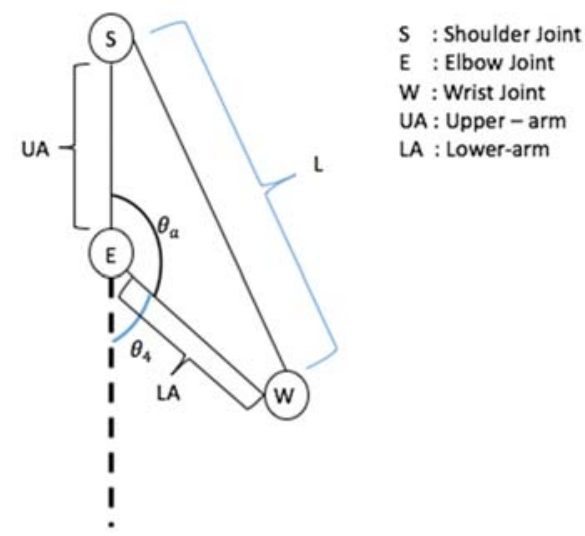

Figure 5. Position of $\theta_{4}$ 
To obtain $\theta_{1}$, information from forward kinematics is used, which is a position of $\mathrm{x}$, at Joint 1 , and it is represented as:

$$
x_{1}=L_{2} \cos \theta_{1}
$$

By rearranging $(6), \theta_{1}$ is obtained as :

$$
\pm \theta_{1}=\cos ^{-1} \frac{x_{1}}{L_{2}}
$$

Note that $\theta_{1}$ is restricted to $[0, \pi]$, hence the possible values exist in Quadrant I and Quadrant II. A condition statement is applied to obtain the final value of $\theta_{1}$ in (7). The condition statement is

\begin{tabular}{|c|c|}
\hline No & Algorithm \\
\hline 1. & If input $\geq 0$ \\
\hline 2. & $\theta_{1}=\theta_{1}$ \\
\hline 3. & Else \\
\hline 4. & $\theta_{1}=-\theta_{1}$ \\
\hline
\end{tabular}
shown in Table 4.

in the next section, the proposed control mechanism of an upper-limb exoskeleton is presented.

\subsection{Control mechanism of an upper-limb exoskeleton}

The general structure of the human-exoskeleton system is shown in Figure 6. The exoskeleton will receive two pieces of information: desired trajectory and level of human strength. The exoskeleton needs to know the level of human strength because it will send assistance as much as human needs.

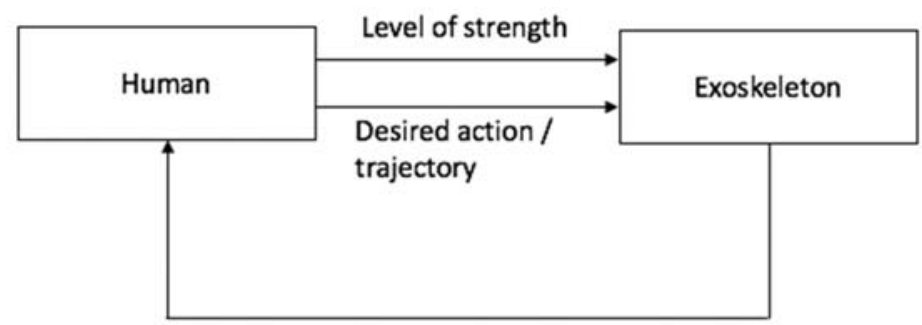

Level of support / assistance

Figure 6. Structure of human-exoskeleton system

The block diagram of Figure 6 is presented in Figure 7. In a real world, the orientation or the desired action of the shoulder and elbow are known to human. The $a_{1}$ and $b_{1}$ represent the strength progression of the human shoulder and elbow joints. Meanwhile, the $\mathrm{a}_{2}$ and $\mathrm{b}_{2}$ represent the percentage needed to assist human.

In this work, the exoskeleton is assumed to assist human from the beginning of the movement. The purpose is to ensure that the proposed control approach could be used to assist human with less strength. The experiment is done in one joint movement; shoulder flexion/extension. Hence, in this work, the gain $b_{1}$ and $b_{2}$ is none. Three conditions of human strength of shoulder flexion-extension is shown in Table 5.

Table 5. Three conditions: Single joint

\begin{tabular}{ccc}
\hline Human strength & a1 & a2 \\
\hline Condition 1 & $100 \%$ & $0 \%$ \\
Condition 2 & $70 \%$ & $0 \%$ \\
Condition 3 & $70 \%$ & $30 \%$ \\
\hline
\end{tabular}


In this work, the PID and extended-fuzzy controllers are investigated. In the next section, both controllers are explained.

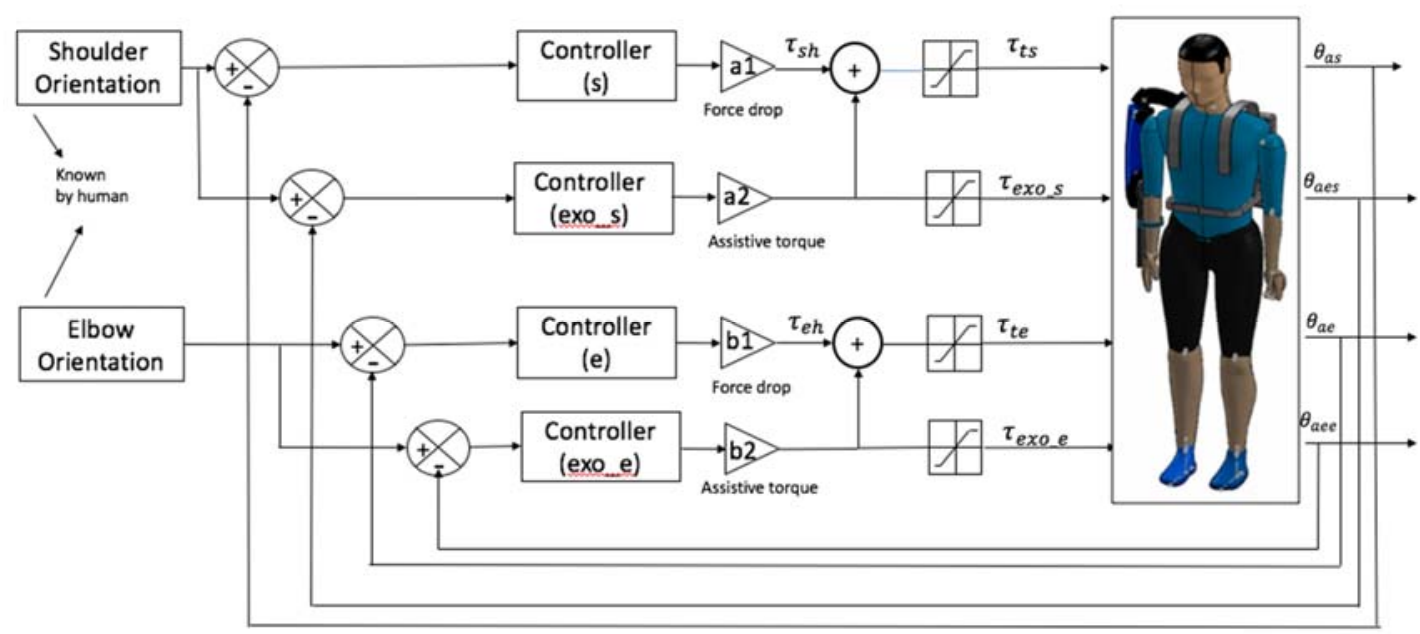

Figure 7. Simulink control diagram of shoulder and elbow joints of human and exoskeleton

\subsection{Implementation of the controller: Proportional-Integral-Derivative (PID) control}

The PID control is used as a baseline for comparison purpose, because of the simplicity in implementation and reliability. In addition, PID control is easy to tune to provide robust performance in an application, and it does not require mathematical model of the system. The block diagram for shoulder flexionextension is shown in Figure 8.

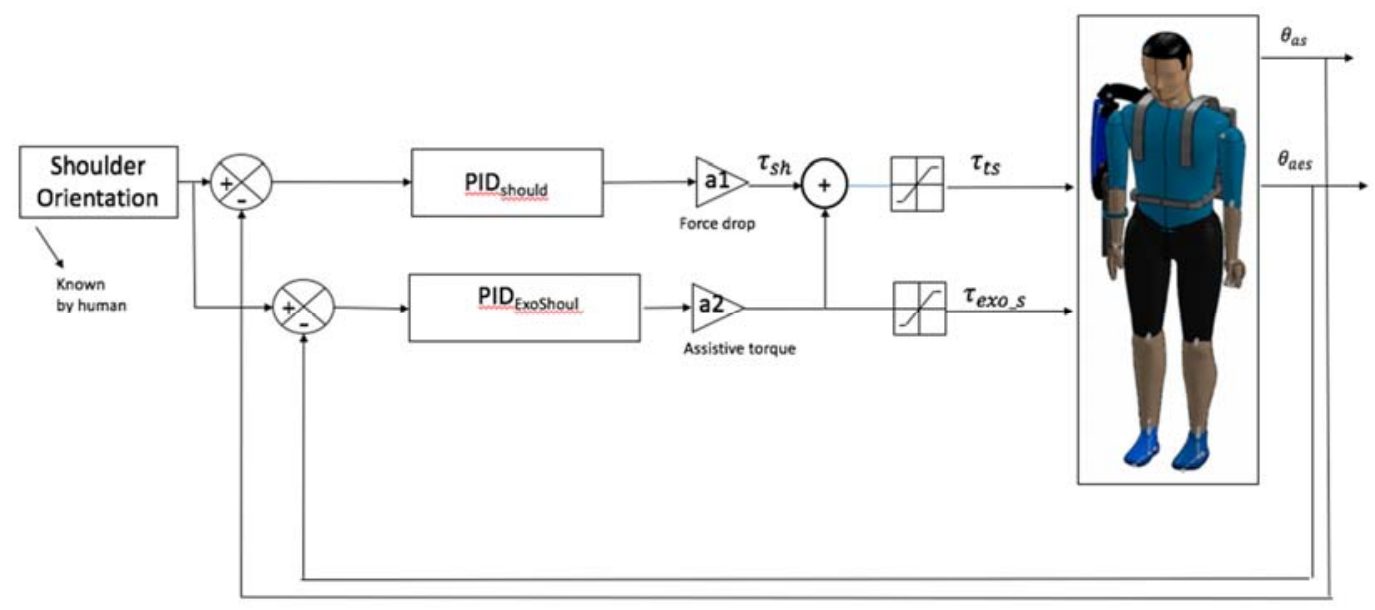

Figure 8. Block diagram of the PID

The input to the PID control is the tracking error $\left(\theta_{e}\right)$. The tracking error is obtained by subtracting the desired trajectory from the actual trajectory of the exoskeleton. Then, based on the control inputs, the controller generates the control signal. The control signal is fed to the plant. The output from the plant is fed back and compared to the reference. This process is continuously occurred until the desired reference is achieved. 


\subsection{Implementation of the controller: Extended-based fuzzy control}

Since the experiment is done in a virtual platform, hence, the human is controlled by using fuzzybased PD control, and the exoskeleton is controlled by using extended-based fuzzy control. Block diagram of the extended-based fuzzy control as shown in Figure 9. The extended-based fuzzy control is consists of fuzzybased motion and fuzzy-based de-weight Figure 10.

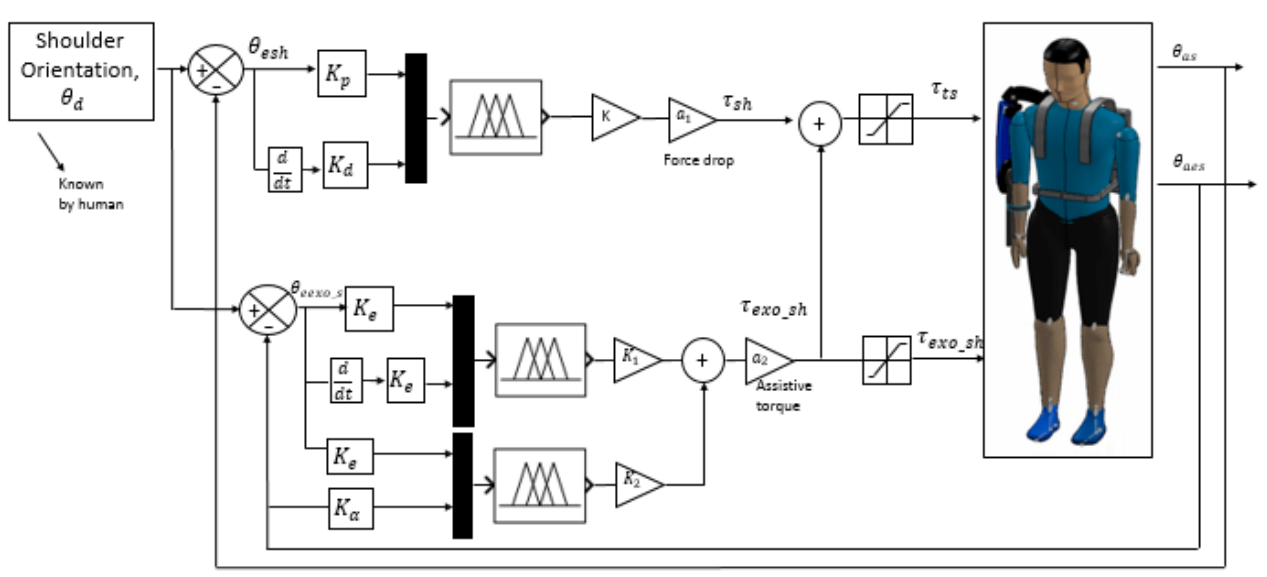

Figure 9. Block diagram of the extended-based fuzzy control

The inputs to the fuzzy-based motion are tracking error $\left(\theta_{e}\right)$ and the rate of the change of the tracking error $\left(\dot{\theta}_{e}\right)$. Meanwhile, the inputs to the fuzzy-based de-weight are the tracking error $\left(\theta_{e}\right)$ and the current position of the joint $\left(\theta_{a}\right)$. The tracking error is obtained by subtracting the desired trajectory from the actual trajectory of the exoskeleton. The controller, will generated control signal based on the control inputs. For fuzzy, the control signal generates from based-motion and based-de-weight, is accumulated. This control signal, is then, added to the control signal, generates from human, and is sent to human. The force drop is assumed to be human strength. The assistive torque block, is the assistive torque by exoskeleton to assist human when the strength is low. The output from the plant is fed back and is compared to the set-point. This process will continuously occur until the set-point is achieved.

There are five membership functions (MFs) used in modeling the fuzzy-based motion and fuzzy-based-deweight: Negative Big (NB), Negative Small (NS), Zero (Z), Positive Small (PS), Positive Big (PB). This membership function was normalized in the range of $[-1,1]$. Each fuzzy controller was $50 \%$ overlap between MFs. The inference mechanism for both fuzzy is max-min method and defuzzification approach is the centroid of gravity (COG). Construction of fuzzy rules: Fuzzy-based motion as shown in Table 6. The details of MFs is presented in Figures 10 and 11.

Table 6. Construction of fuzzy rules: Fuzzy-based motion

\begin{tabular}{llllll}
\hline$\theta_{e} / \dot{\theta}_{e}$ & $\mathrm{NB}$ & $\mathrm{NS}$ & $\mathrm{Z}$ & $\mathrm{PS}$ & $\mathrm{PB}$ \\
\hline $\mathrm{NB}$ & $\mathrm{PB}$ & $\mathrm{PB}$ & $\mathrm{PB}$ & $\mathrm{PS}$ & $\mathrm{Z}$ \\
$\mathrm{NS}$ & $\mathrm{PB}$ & $\mathrm{PB}$ & $\mathrm{PS}$ & $\mathrm{Z}$ & $\mathrm{NS}$ \\
$\mathrm{Z}$ & $\mathrm{PB}$ & $\mathrm{PS}$ & $\mathrm{Z}$ & $\mathrm{NS}$ & $\mathrm{NB}$ \\
$\mathrm{PS}$ & $\mathrm{PS}$ & $\mathrm{Z}$ & $\mathrm{NS}$ & $\mathrm{NB}$ & $\mathrm{NB}$ \\
$\mathrm{PB}$ & $\mathrm{Z}$ & $\mathrm{NB}$ & $\mathrm{NB}$ & $\mathrm{NB}$ & $\mathrm{NB}$ \\
\hline
\end{tabular}

Indo. J. Elec. Eng. \& Inf, Vol. 7, No. 1, March 2019: 1 - 14 


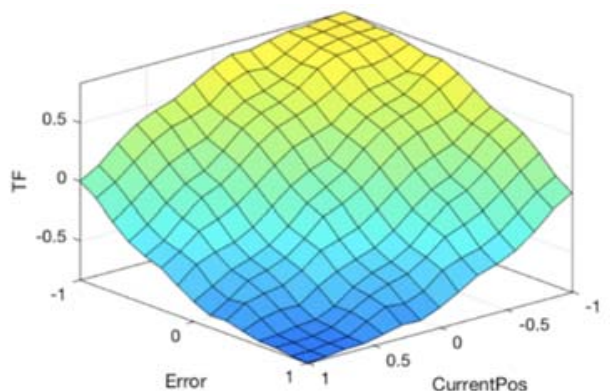

(a1)

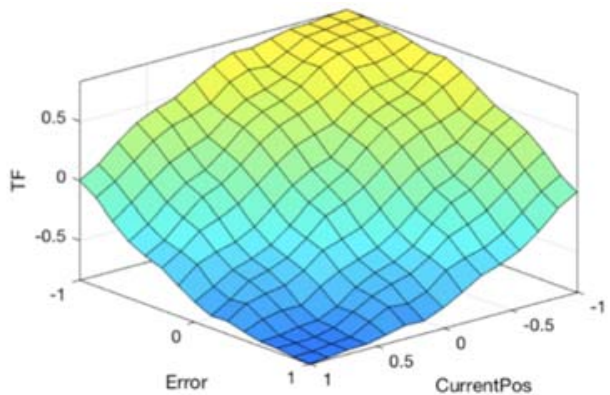

(b1)

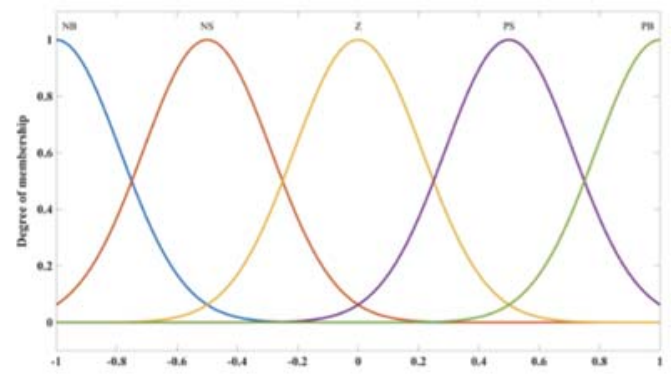

(a2)

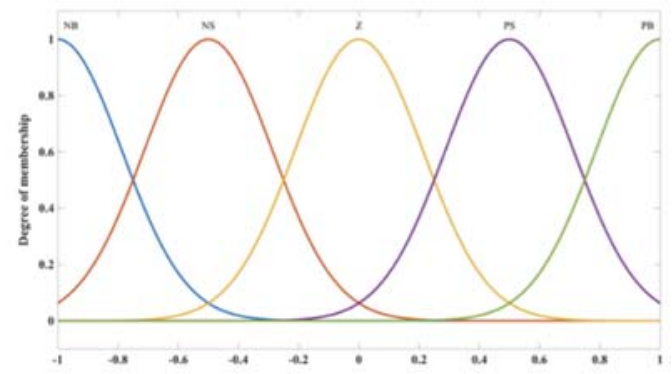

(b2)

Figure 10. Details of fuzzy-based motion: (a1) Fuzzy 3D surface (a2) Membership functions for inputs and outputs. Detail of fuzzy-based de-weight control: (b1) Fuzzy 3D surface (b2) Membership functions for inputs and outputs

Table 7. Construction of fuzzy rules: Fuzzy-based de-weight

\begin{tabular}{cccccc}
\hline$\theta_{e} / \alpha_{e}$ & $\mathrm{NB}$ & $\mathrm{NS}$ & $\mathrm{Z}$ & $\mathrm{PS}$ & $\mathrm{PB}$ \\
\hline $\mathrm{NB}$ & $\mathrm{PB}$ & $\mathrm{PB}$ & $\mathrm{PB}$ & $\mathrm{PS}$ & $\mathrm{Z}$ \\
$\mathrm{NS}$ & $\mathrm{PB}$ & $\mathrm{PB}$ & $\mathrm{PS}$ & $\mathrm{Z}$ & $\mathrm{NS}$ \\
$\mathrm{Z}$ & $\mathrm{PB}$ & $\mathrm{PS}$ & $\mathrm{Z}$ & $\mathrm{NS}$ & $\mathrm{NB}$ \\
$\mathrm{PS}$ & $\mathrm{PS}$ & $\mathrm{Z}$ & $\mathrm{NS}$ & $\mathrm{NB}$ & $\mathrm{NB}$ \\
$\mathrm{PB}$ & $\mathrm{Z}$ & $\mathrm{NB}$ & $\mathrm{NB}$ & $\mathrm{NB}$ & $\mathrm{NB}$ \\
\hline
\end{tabular}

The rules for both fuzzy were developed based on the knowledge to minimize the steady-state error and rise-time to ensure the power consumption is less, and to ensure that the joints can move to the desires trajectory accurately.

In both system, saturation is included to ensure that the control signal is in the range of the human torque capacity.

Table 8. Torque limits of human arm [26]

\begin{tabular}{l|l}
\hline Joint & Human strength $(\mathrm{Nm})$ \\
\hline Shoulder flexion-extension & $115 / 110$ \\
Shoulder abduction-adduction & $134 / 94$ \\
Elbow flexion-extension & $72.5 / 42$ \\
Forearm pronation-supination & $9.1 / 7$ \\
Wrist palmer-dorsal flexion & 19.8 \\
Wrist abduction-adduction & 20.8 \\
\hline
\end{tabular}

\section{RESULTS AND ANALYSIS}

In this section, the results and discussion on the performance of PID and extended based fuzzy control are presented. There are three categories of the performance: trajectory tracking, error performance and torque required by both controllers. This section begins with results of PID and follows with extended based fuzzy controller.

Control design of a de-weighting upper-limb exoskeleton: extended-based fuzzy (Siti Khadijah Ali) 
In this experiment, only shoulder joint is involved Figure 11. The shoulder joint is moving in flexionextension movement. The remaining joints are assumed to be passive. The human strength conditions and assistive torque is presented as in Table 9. In this experiment, there are three conditions of human strength. First, the shoulder joint has $100 \%$ strength, hence, no need to be assisted by the exoskeleton. In second case, the shoulder joint is assumed to have $70 \%$ strength, and in this case, no assistance from exoskeleton. Third case, the human strength is limited to $70 \%$ strength, and exoskeleton is assist the remaining $30 \%$.

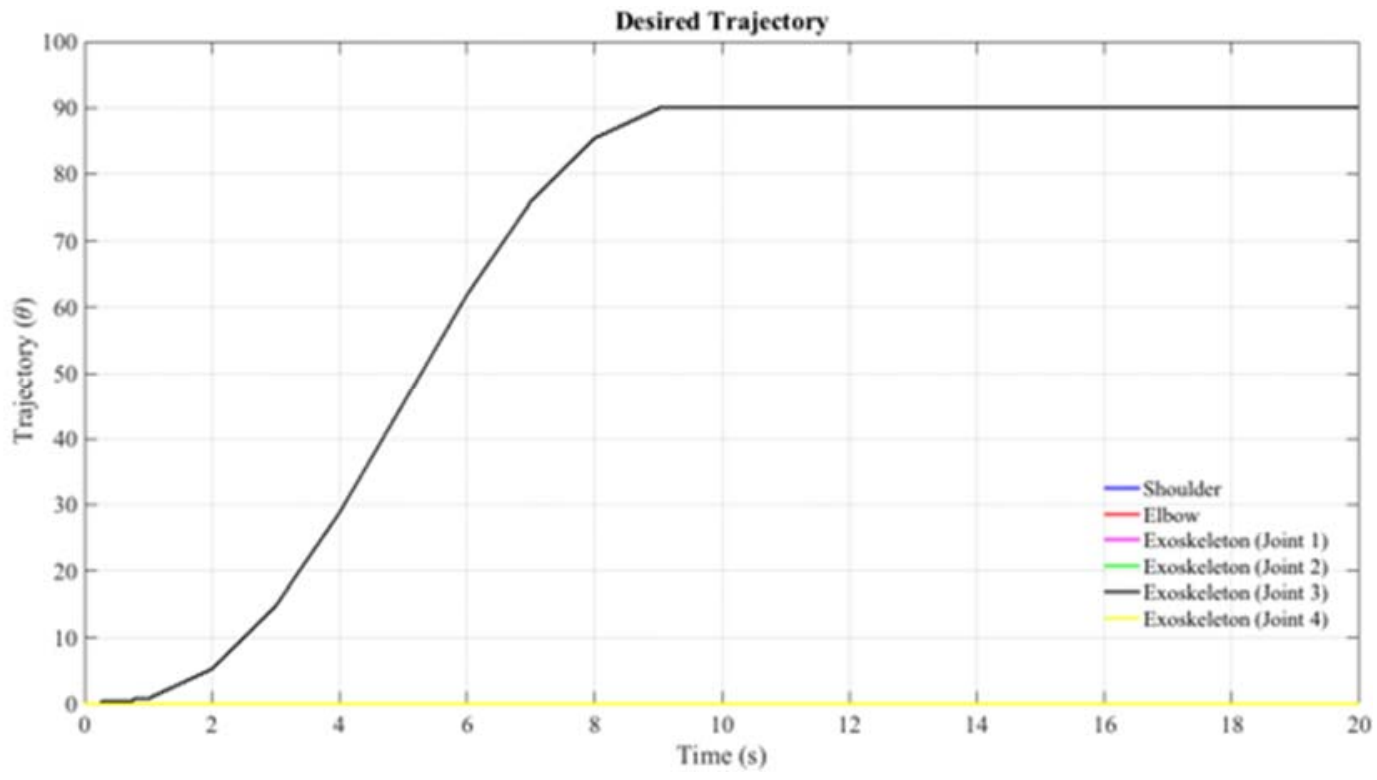

Figure 11. Desired trajectory

Table 9. Three conditions: Single joint

\begin{tabular}{ccc}
\hline Case & a1 (Human strength) & a2 (Assistive torque) \\
\hline Condition 1 & $100 \%$ & $0 \%$ \\
Condition 2 & $70 \%$ & $0 \%$ \\
Condition 3 & $70 \%$ & $30 \%$ \\
\hline
\end{tabular}

For both controllers, the gains were obtained in a heuristic approach. Table 10 shows the gains used in this experiment.

Three different measurements were used to analyses the performance of the control approach: Root Mean Square Error (RMSE), Maximum Absolute Error (MAE) and Maximum Absolute Torque (MAT). The formula for both calculations are:

$$
\begin{aligned}
& R M S E=\sqrt{\frac{\sum_{i=1}^{n}\left(\theta-\theta_{a}\right)^{2}}{n}} \\
& M A T=\max \left(\left|\theta-\theta_{a}\right|\right) \\
& M A T=\max (|\tau|)
\end{aligned}
$$

where $\theta$ is the desired trajectory and $\theta_{a}$ is the actual trajectory; $\mathrm{n}$ is the number of data.

Table 10. Control gains: PID controller

\begin{tabular}{ccc}
\hline Gains & Shoulder joint & Exoskeleton (Joint 3) \\
\hline$K_{p}$ & 10 & 10 \\
$K_{i}$ & 1 & 1 \\
$K_{d}$ & 0.1 & 0.1 \\
\hline
\end{tabular}


Generally, as noted, in all conditions human could achieve the desired trajectory. However, in Figure 12 (b) and (c) show an active fluctuation. This occur due to graphical issue of the signal builder and could not be avoided Figure 13, but the range is considered as small $\left(\leq 1^{0}\right)$. The active actuations, however, disappear after approximately 10 seconds. This is because, the difference between current position to the next position of the joint is $0^{0}$ (no joint movement) from second 10 to second 20.
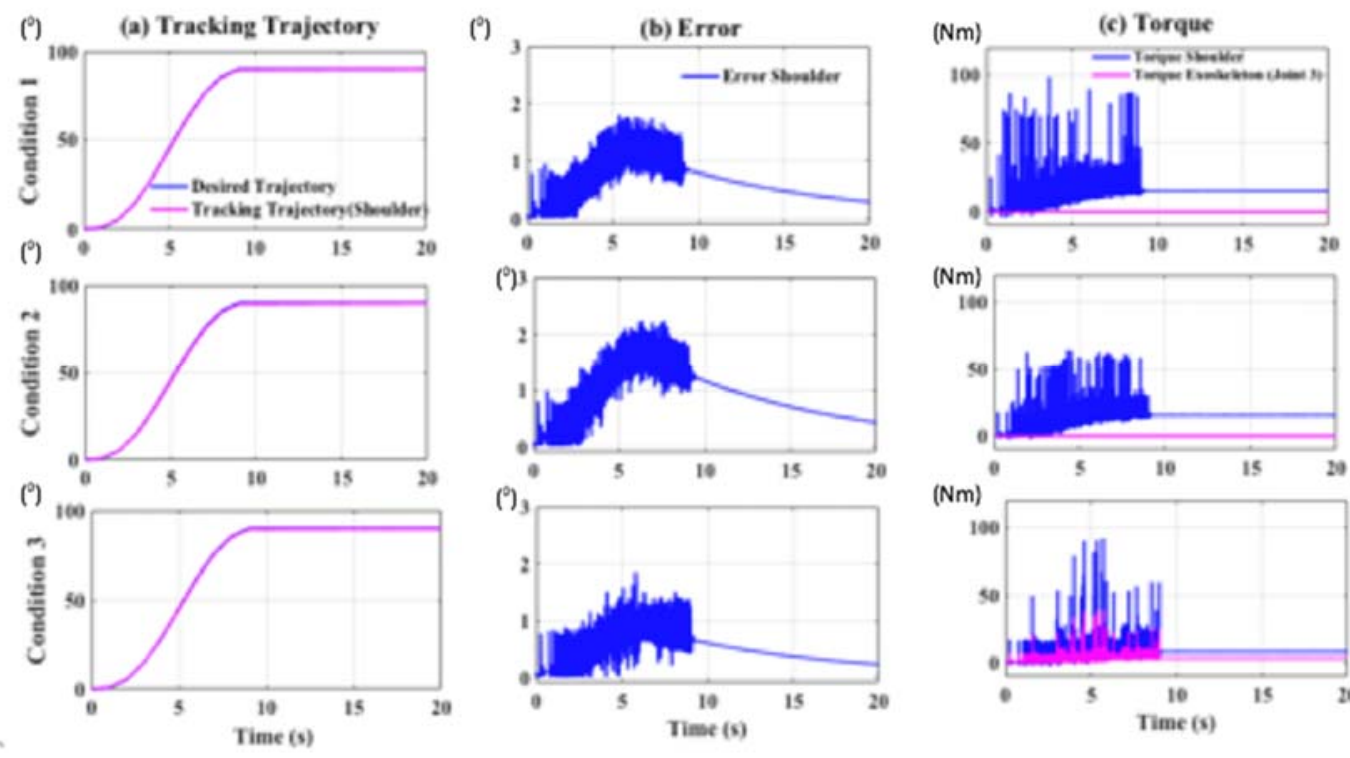

$(\mathrm{Nm})$

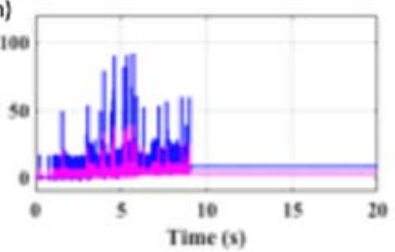

Figure 12. Results of PID control: (a) Trajectory tracking (b) Error (c) Torque

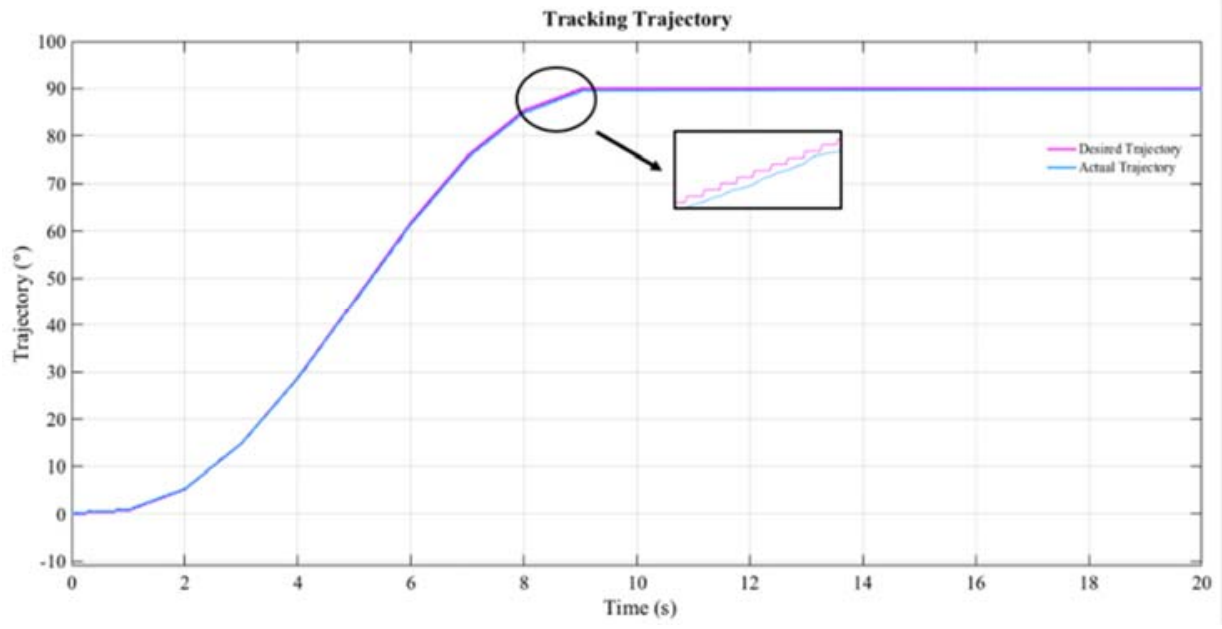

Figure 13. Graphical issue of signal builder

It is obvious that the third condition shows the least error compared to the first and second condition, where the MAE is about $1.80^{\circ}$. The RMSE results show the similar trend as MAE.

To observe the torque required for this movement, a torque required to maintain the shoulder joint at the static position is chosen because human is easily to get fatigue at this position. At this position, the MAT values need by shoulder joint at first and second conditions is nearly to $15.4 \mathrm{Nm}$. Whereas, in the third condition, the required torque for shoulder to move was only $11.9 \mathrm{Nm}$ and the torque supplied by the exoskeleton with PID control was $3.55 \mathrm{Nm}$.

Control design of a de-weighting upper-limb exoskeleton: extended-based fuzzy (Siti Khadijah Ali) 
Based on the error and torque analysis, it shows that the torque needed by human to move the upperlimb and exoskeleton is less with the assisted torque from the exoskeleton. This observation is potentially capable of reducing the chances of human muscles from fatigue.

Table 11 shows the gains for the extended-based fuzzy control and Figure 14 shows the results of the extended based fuzzy for all conditions.

Table 11. Control gains: Extended-based fuzzy controller

\begin{tabular}{ccc}
\hline Gains & Shoulder joint & Exoskeleton (Joint 3) \\
\hline$K_{e}$ & 1 & 1 \\
$K_{\dot{e}}$ & 0.001 & 0.001 \\
$K_{\text {gain } 1}$ & 50 & 50 \\
$K_{e}$ & - & 1 \\
$K_{a}$ & - & 0.001 \\
$K_{\text {gain } 2}$ & - & 10 \\
\hline
\end{tabular}

(a) Tracking Trajectory $\left({ }^{\circ}\right)$
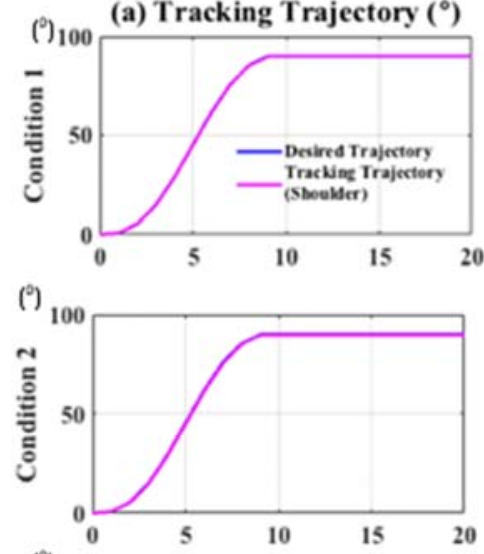

( $\left.{ }^{\circ}\right)$

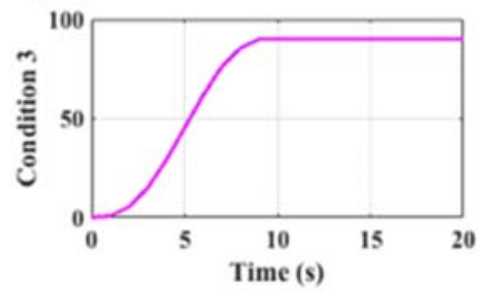

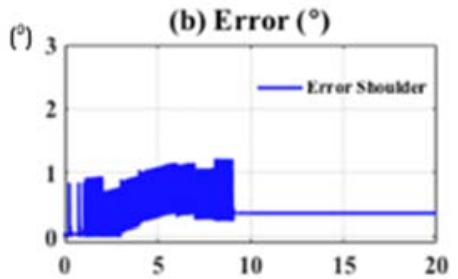

$\left({ }^{\circ}\right)$

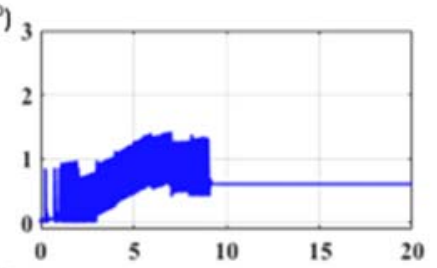

( ${ }^{\circ}$

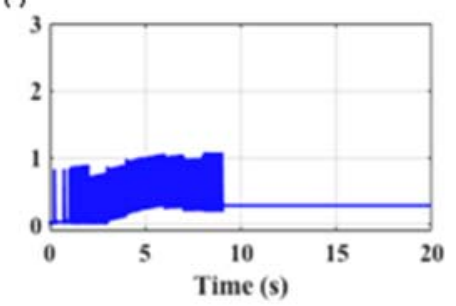

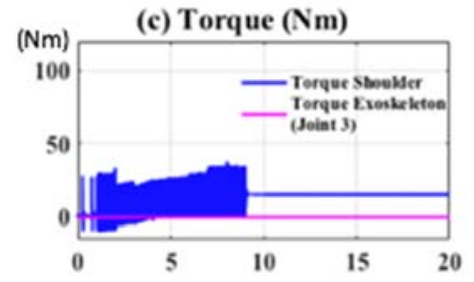

$(\mathrm{Nm})$
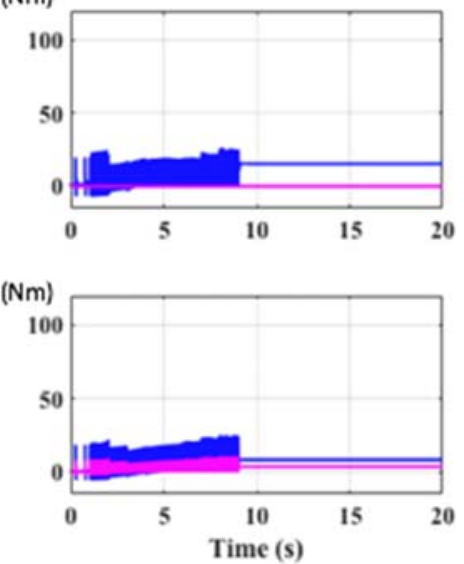

Figure 14. Results of extended-based fuzzy control: (a) Trajectory tracking (b) Error (c) Torque

It is noted that, in general, the exoskeleton with extended-based fuzzy controller was able to control the movements of the upper-limb in all conditions. Similar as PID controller, an active fluctuation could be observed occurs in Figure 14 (b) and (c), which occur due to graphical issue of the signal builder. It is observed that, the error of the third condition is the least compared to the first and second conditions, and the highest MAE was the second condition.

Table 12 shows the exact values in terms of RMSE and MAE for both controllers. It is clearly shown that the third condition for both controllers show the least error, and the second condition show the highest errors amongst three conditions. This shows that the, with the help of exoskeleton, human could achieve the desired trajectory accurately. In addition, the RMSE values with PID control for all conditions were higher to nearly $50 \%$ than extended based fuzzy control. The MAE values were similarly $50 \%$ higher with PID control in comparison to those with extended-based fuzzy. Hence in deviation observation, the extended-based fuzzy, showed the better performance compared to PID control. 
Table 12. RMSE and MAE for PID and extended-based fuzzy controllers

\begin{tabular}{ccccc}
\hline Case & \multicolumn{2}{c}{ RMSE } & \multicolumn{2}{c}{ MAE } \\
& PID & Extended-based fuzzy & PID & Extended-based Fuzzy \\
\hline Condition 1 & 0.7829 & 0.4911 & 1.848 & 1.208 \\
Condition 2 & 1.061 & 0.5809 & 2.236 & 1.432 \\
Condition 3 & 0.6229 & 0.4294 & 1.80 & 1.052 \\
\hline
\end{tabular}

Table 13. MAT for PID and extended-based fuzzy controllers

\begin{tabular}{|c|c|c|c|c|}
\hline \multirow[t]{2}{*}{ Case } & \multicolumn{2}{|r|}{ Shoulder } & \multicolumn{2}{|c|}{ Exoskeleton (Joint 3) } \\
\hline & PID & Extended-based fuzzy & PID & Extended-based Fuzzy \\
\hline Condition 1 & 15.4 & 15.45 & 0 & 0 \\
\hline Condition 2 & 15.4 & 15.45 & 0 & 0 \\
\hline Condition 3 & 11.9 & 7.612 & 3.55 & 3.915 \\
\hline
\end{tabular}

The values of the MAT for the controllers are shown in Table 13. It is noted that the torque required by the exoskeleton, with extended-based fuzzy control assisting the human was about $29 \%$ higher than with the PID control. However, in terms of trajectory tracking error performance, extended-based fuzzy controller performed nearly 50\% better compared to PID control. Moreover, as in addition, Figure 14 (c) shows that the torque fluctuated in an acceptable range for human shoulder and the exoskeleton joint. These show that by implementing an intelligent control approach such as fuzzy logic control, the required output can be obtained to an acceptable range, hence could limit the potential of damage or harm to the whole system.

\section{CONCLUSION}

This study proposed a control design-based extended fuzzy to ensure the upper-limb exoskeleton could moves accurately to a desired position. The extended-based fuzzy consists of fuzzy-based motion and fuzzy-based de-weight. Three different conditions of human are included, to investigate the effectiveness of the proposed control approach. It is shown that the extended-based fuzzy, can assists human to the desired position, accurately, with a minimal torque required. Hence in future, this control approach will be implemented to a human with the inclusion of fatigue model.

\section{ACKNOWLEDGEMENT}

This research was supported financially by the Ministry of Higher Education, Universiti Putra Malaysia and University of Sheffield.

\section{REFERENCES}

[1] Fan L, Yan L, Xiao J, Wang F., "Dynamics analysis and simulation verification of a novel knee joint exoskeleton," Journal of Vibroengineering, vol. 19(4), 2017.

[2] Garrec P., "Design of an anthropomorphic upper limb exoskeleton actuated by ball-screws and cables," Bulletin of the Academy of Sciences of the Ussr-Physical Series, vol. 72(2), pp. 23, 2010.

[3] Ma L, Bennis F, Chablat D, Zhang W., "Framework for dynamic evaluation of muscle fatigue in manual handling work," in Industrial Technology, ICIT 2008. IEEE International Conference on 2008, pp. 1-6, IEEE, 2008.

[4] Ma L, Chablat D, Bennis F, Zhang W., "A new simple dynamic muscle fatigue model and its validation," International Journal of Industrial Ergonomics, vol. 39(1), pp. 211-20, 2009.

[5] Ma R, Chablat D, Bennis F., "A new approach to muscle fatigue evaluation for Push/Pull task," in Romansy 19-Robot Design, Dynamics and Control, Springer, Vienna, pp. 309-316, 2013.

[6] Sakka S, Chablat D, Ma R, Bennis F., "Predictive model of the human muscle fatigue: application to repetitive pushpull tasks with light external load," arXiv preprint arXiv:1503.06391.2015.

[7] Seth D, Chablat D, Bennis F, Sakka S, Jubeau M, Nordez A., "Validation of a new dynamic muscle fatigue model and dmet analysis," The International Journal of Virtual Reality, vol. 2016(16), 2016.

[8] Pearce N, Dryson E, Feyer AM, Gander P, McCracken S, Wagstaffe M., "Burden of occupational disease and injury in New Zealand: report to the Associate Minister of Labour," In Burden of occupational disease and injury in New Zealand: report to the Associate Minister of Labour 2004. National Occupational Health and Safety Advisory Committee, 2004.

[9] Abelson, Brian, and Kamali T. Abelson, "Release your pain: Resolving repetitive strain injuries with active release techniques," Rowan Tree Books, 2004

[10] Giat Y, Mizrahi J, Levy M., "A musculotendon model of the fatigue profiles of paralyzed quadriceps muscle under FES," IEEE transactions on biomedical engineering, vol.40(7), pp. 664-74, 1993.

[11] Liu JZ, Brown RW, Yue GH., "A dynamical model of muscle activation, fatigue, and recovery," Biophysical journal. vol. 82(5), pp. 2344-59, 2002.

Control design of a de-weighting upper-limb exoskeleton: extended-based fuzzy (Siti Khadijah Ali) 
[12] Ma L, Chablat D, Bennis F, Zhang W, Hu B, Guillaume F., "A novel approach for determining fatigue resistances of different muscle groups in static cases," International Journal of Industrial Ergonomics, vol. 41(1), pp.10-8, 2011.

[13] Ma L, Chablat D, Bennis F, Zhang W, Guillaume F., "A new muscle fatigue and recovery model and its ergonomics application in human simulation," Virtual and Physical Prototyping, vol. 5(3), pp.123-37, 2010.

[14] Ma R, Chablat D, Bennis F, Ma L. "Human muscle fatigue model in dynamic motions," In Latest Advances in Robot Kinematics. Springer, pp. 349-356, 2012.

[15] Borzelli D, Pastorelli S, Gastaldi L., "Elbow musculoskeletal model for industrial exoskeleton with modulated impedance based on operator's arm stiffness," Int. J. of Automation Technology, vol. 11(3), 2017.

[16] Bosch T, van Eck J, Knitel K, de Looze M., "The effects of a passive exoskeleton on muscle activity, discomfort and endurance time in forward bending work," Applied ergonomics, vol. 54, pp. 212-7, 2016.

[17] Xu W, Chu B, Rogers E., "Iterative learning control for robotic-assisted upper limb stroke rehabilitation in the presence of muscle fatigue," Control Engineering Practice, vol. 31, pp. 63-72, 2014.

[18] Sylla N, Bonnet V, Colledani F, Fraisse P., "Ergonomic contribution of ABLE exoskeleton in automotive industry," International Journal of Industrial Ergonomics," vol. 44(4), pp. 475-81, 2014.

[19] Ma L, Chablat D, Bennis F, Zhang W, Guillaume F., "A new muscle fatigue and recovery model and its ergonomics application in human simulation," Virtual and Physical Prototypin, vol. 5(3), pp. 123-37, 2010.

[20] Ren Y, Kang SH, Park HS, Wu YN, Zhang LQ., "Developing a multi-joint upper limb exoskeleton robot for diagnosis, therapy, and outcome evaluation in neurorehabilitation," IEEE Transactions on Neural Systems and Rehabilitation Engineering, vol. 21(3), pp. 490-9, 2013.

[21] Gopura RARC, Kiguchi K, Yi Y., "SUEFUL-7: a 7DOF upper-limb exoskeleton robot with muscle-model-oriented EMG-based control," in: IEEE/RSJ international conference on i ntelligent robots and systems, pp. 1126-31, 2009.

[22] Carignan C, Tang J, Roderick S., "Development of an exoskeleton haptic interface for virtual task training," In: IEEE/RSJ international conference on intelligent robots and systems, pp. 3697-702, 2009.

[23] Nef T, Guidali M, Riener R., "ARMin III—arm therapy exoskeleton with an ergonomic shoulder actuation," Applied Bionics and Biomechanics, pp. 127-42, 2009.

[24] Rahman MH, Kiguchi K, Rahman MM, Sasaki M., "Robotic exoskeleton for reha- bilitation and motion assist," In: International conference on industrial and information systems, pp. 241-6, 2006.

[25] Luijten F, Chu B, Rogers E., "Iterative learning control for stroke rehabilitation with input dependent muscle fatigue modeling," In 2018 Annual American Control Conference (ACC), pp. 6396-6401, IEEE, 2018.

[26] Głowiński S, Krzyżyński T, Pecolt S, Maciejewski I., "Design of motion trajectory of an arm exoskeleton," Archive of Applied Mechanics, vol. 85(1), pp. 75-87, 2015.

Indo. J. Elec. Eng. \& Inf, Vol. 7, No. 1, March 2019: 1 - 14 